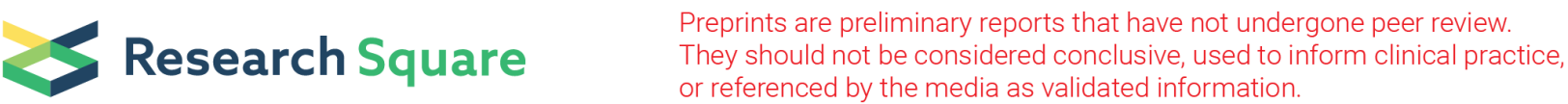

\section{The Use of the Artificial Neural Network for the Treatment Outcomes of Single-channel and Tri- channel Applicator Used in Cervical Cancer Based on High Dose Rate Brachytherapy}

Alamgir Hossain ( $\nabla$ hossain447a@gmail.com )

University of Rajshahi

Shahidul Miah

Rajshahi Medical College

Prodip Kumer Ray

Rajshahi Medical College

Ashim Kumar Ghosh

Rajshahi Medical College

Rawshan Ara Khatun

Rajshahi Medical College

Julekha Sarker

Rajshahi Medical College

Shafayat Habib

Rajshahi Medical College

Shupti Sarker

University of Rajshahi

\section{Research Article}

Keywords: Artificial neural network, treatment outcomes, single-channel applicator, tri-channel applicator, high dose rate brachytherapy, cervical cancer

Posted Date: June 7th, 2021

DOI: https://doi.org/10.21203/rs.3.rs-556879/v1

License: (c) (i) This work is licensed under a Creative Commons Attribution 4.0 International License.

Read Full License 


\section{Abstract}

The study aimed to evaluate the treatment outcomes of single-channel and tri-channel applicators for cervical cancer patients based on high dose rate brachytherapy using an artificial neural network. An artificial neural network (ANNs) model is proposed to predict the treatment outcomes for the singlechannel applicator and tri-channel applicator in cervical cancer for high dose rate brachytherapy. Fiftyfour patients of cervical cancer who were receiving external beam radiation therapy (EBRT) of 40-50 cGy, with chemotherapy, were selected in this study from 37 patients with cervical cancer being used to train and 17 for testing in this model. A model was developed for intracavitary brachytherapy to estimate the comparison of treatment outcomes for the single-channel applicator and tri-channel applicators, demonstrating the sensitivity $100 \%$ and specificity $100 \%$ and accuracy $100 \%$ for training and $87.5 \%$, $77.8 \%$, and $82.4 \%$ for testing, respectively, including AUC $=0.961$. The survival rate was $85 \%$ and $95 \%$ for single-channel and tri-channel applicators at 2 years, respectively. A model approach for artificial neural networks based on gynecological brachytherapy is a promising method for patient's treatment, resulting in the dosimetry output of applicators; medical physicists can be decided the appropriate applicator for cervical cancer. The proposed model has the potential accuracy in judging the treatment outcomes for the single-channel applicator and tri-channel applicator in cervical cancer based on survival analysis.

\section{Introduction}

In the world, cervical cancer is the top most cancer. It is ranked fourteen among all cancers and fourth position for women in the world.[1] In Western Africa, the most frequent cancer is cervical for women. Papillomavirus infection, sex partners, economic status, and smoking are the risk factors for cervical cancer.[2, 3] According to the statistics 2012, the incident rate of $1.3 \%$ has decreased in 2015[4], due to improvement of lifestyle, vaccination, and early detection.[5] Still now, it is the highest mortality in Asia, counting in the $53.8 \%$ of total cancer cases.[6]

The treatment type of cervical cancers is generally intracavitary, interstitial, and hybrid. The American Brachytherapy Society has recommended cervical cancer patients to treat the symptoms such as vaginal extension, pelvic sidewall and bulky lesions, and vaginal apex.[7] Although the single-channel applicator is used for the non-bulky diseases to avoid the dose from the organ at risk (OAR) like rectum and bladder, multi-channel is also used to deliver dose decreased in OARs by the dwell time of the applicators. The other literature has discussed that a single channel reduces cost, coverage for covering doses in tumors and decreases doses in OARs than multi-channel applicators.[8] In the further article showed that a multi-channel applicator could reduce tumor dose and rectum dose as single-channel applicators with statistically significant, resulting in favor of a multi-channel applicator when a single channel inserted at $5 \mathrm{~mm}$ prescription at depth.[9]

Traditionally, logistic regression (LR) is used worldwide to predict the developed models and analyze medical outcomes.[10] However, there is some limitation based on the ability to predict the model with accuracy. For example, LR is the relationship between independent and dependent variables, 
demonstrating the normal distribution of independent variables.[11] Therefore, modeling complex systems like treatment outcomes of a single-channel applicator and tri-channel applicator, LR is not appropriate for capturing the complex relationship and finding the accuracy among the independent variables such as treatment outcomes.

In contrast to LR, ANN is a type of artificial intelligence, demonstrating problem-solving methods in medicine and biomedical engineering enormously worldwide.[12] It is used in biomedical science, drug development, treatment, health care, and medical image processing. ANNs are the model for information processing that describes the interconnected neurons resolving the problems.[13] Provided that it is trained the ANNs to generalize, it will be suitable to predict results quickly other than training.[14] In ANNs is used based on a backpropagation algorithm.[15] A training set is feed to ANNs for processing the input and output data to learn. The output is compared to human-provided output. If the prediction is not correct, the algorithm learns through the backpropagation methods to adjust the mathematical equation for minimizing the errors in the training period.[16]

In volumetric dose analysis, Rajković et al. proposed the artificial neural network with a genetic algorithm to optimize brachytherapy parameters.[17] An ANNs model was developed for intra-factional OARs dose in Jaberi et al., describing applicator changes to compensate the treatment plan[18] and is also used in business, credit and fraud detection, speech recognition, and image processing.[19] The model was applied for traumatic brain injury[20], the lumbar spine canal stenosis[21], cancer identification with outcomes[22], detection of posterior lumbar spine fusion[23], and the diagnosis of my cardinal complexation.[24] The ANNs have unique benefits such as decision making, improving treatment outcomes, and reduce treatment cost.[16] The treatment outcomes are considered based on the patient's last follow-up after treatments and the survival rate for the applicators used in cervical cancer. Therefore, we have developed a model to predict the treatment outcomes based on survival analysis of singlechannel and tri-channel applicators used in cervical cancer.

\section{Materials And Methods}

\section{Patients preparation}

The prospective study was designed of 54 patients (training: 37, testing: 17) with cervical cancer with stages $1 \mathrm{~b}$ to IVa, who underwent admission at our Medical college hospital from 2015 to 2020. Approval from the institutional ethical committee was taken. Patients record data used for this study. After chemotherapy of cisplatin, the patients were treated 45-50 Gy of EBRT at 21 frication with high dose tare brachytherapy, three fractions at a one-week interval. We used two Fletcher applicators, including tandem and Ovid, such as a single type applicator and tri-channel applicator for cervical cancer treatment. We also used pre-anesthesia before general anesthesia when we inserted the applicators in lithotomic position. The bladder points of its ballons were colored with diluted urography of 7cc while a Foley catheter was inserted into a urinary bladder. A gauge is used to immobilize these applicators to protect the rectum and bladder. BEBIG Multisource (Eckert and Ziegler, BEBIG, Germany), which contained the 
source of Co-60, active length $3.5 \mathrm{~mm}$, core diameter of $0.5 \mathrm{~mm}$ with high dose rate brachytherapy, was used to treat all patient. For treatment planning, HDR 3.00 plus (Eckert and Ziegler, BEBIG, Germany) TPS was used. The anterior-posterior and lateral images were collected for each patient using a C-Arm x-ray machine. The applicator's reconstruction was set up in anterior or posterior images, according to ICRU 38 . The dose distribution made for anterior-posterior and lateral in Figs. 1 (a) and (b). The dose distribution was performed for individual patients according to the guidelines. The graphical representation for the treatment plane that axial, sagittal, and coronal planes with isodoses taken in Fig. 2. According to the prescribed dose, the isodose curves adjusted to maintain the rectum and bladder dose for each cervical cancer patient. The prescribed dose $7 \mathrm{~Gy}$ was defined as point $\mathrm{A}$, which is $2 \mathrm{~cm}$ lateral distance from the uterus central canal, and $2 \mathrm{~cm}$ upwards from mucus fornix membrane according to an international protocol ICRU 38.[25] Dose calculation was obtained according to the Report (TG-43) of AAPM Task Group. An ANN model was treated to all predictors using an artificial neural network.

\section{Artificial neural networks for the classification of applicators based on treatment outcomes.}

The whole ANN model is shown in Fig. 3. The ANN model has an input layer, hidden layer, and output layer. The input layers belong to patient age, EBRT, decay factor, Air kerma strength, activity, dose volume, applicator insertion, Ovid length, treatment duration, treatment function, treatment result, alive/dead, prescribed dose, tumor area dose, Manchester B, rectum dose, bladder dose, treatment time, and total reference air kerma (TRAK). The two hidden layers used for the model that is interconnected with neurons. The output layer was a single channel applicator (0) and tri-channel applicator (1). This research aimed to evaluate the treatment outcomes of high-dose brachytherapy on cervical cancer using a single channel and tri-channel applicators. Since input parameters were nineteen and the high data dimension and we have no proper knowledge of treatment outcomes, simple statistical analysis was not sufficient, so we had to apply ANNs to obtain our aims in the current study as multi-layer perceptron architecture.

\section{Description of Multilayer Perception architecture}

The model reveals the synaptic weight, the relative number of trains, and the test was $7: 3$. The hidden layer's number was two, including the activation function of a hyperbolic tangent. The output layer was identity. The type of train was batch including gradient descent optimization algorithm, demonstrating initial learning rate 0.4 , momentum 0.9 , interval center 0 , and interval offset \pm 0.5 . The maximum number of steps without a decrease in error was 5 . The maximum training time was 15 minutes. The maximum number of epochs, the minimum relative change in training error, and the minimum relative change in training error ratio were $100,0.0001$, and 0.001 .

\section{Statistical methods}

The Kaplan-Meier log-rank test was used to compare the survival rate for single-channel and tri-channel applicator. All data were analyzed using SPSS software for Window 10 (Version 21.0; IBM Crop., Armonk, NY, USA). The $\mathrm{P}<0.05$ was considered statistically significant. 


\section{Results}

Table 1 shows the clinical characteristics of cervical cancer. It describes the patients' age, patient number with training, testing, histopathological report for each patient, and cancer staging for squamous cell carcinomas and adenocarcinoma. Table 2 gives a case summary for the artificial neural network, demonstrating the information about the dataset built the ANNs model to evaluate the treatment outcomes for single-channel and tri-channel applicators. The sample of training and testing was $68.50 \%$ and $31.50 \%$. Table 3 shows network information to build up the artificial neural network, the details about the input layer, two hidden layers connected with neurons, and an output layer with activation function was the identity for the neural network. The average patient age was 50 (28-75) years, EBRT(cGy): 45 (40-50); Decay Factor: 5.56 (0.47-0.76); Air Kerma Strength (cGycm²h): 13865.52 (11158.5-57390.0); Activity (Ci): 1.17 (0.99-1.59); Dose Volume $\left(\mathrm{cm}^{3}\right)$ 113.6 (58.2-469.25); Applicator Insertion (cm): 4.78 (2-8); Ovid Length $(\mathrm{mm})$ : 24.72 (15-30); Treatment Duration (days): 13.26 (6-27); Treatment Fraction: 2.63 (1-3); Treatment Results: 0.46 (0-1); Alive/ Dead: 0.09 (0-1); Prescribe Dose (Gy): 6.87 (6-7); Tumor Area Dose (Gy): 6.74 (5.64-7); Manchester B (Gy): 1,85 (1.50-2.65); Rectum Dose (Gy): 3.71 (2.29-5.14); Bladder Dose.(Gy): 2 (1.94-5.83); Treatment Time (min): 19.66 (8.52-31.27); and TRAK (cGy.m²): $0.43(0.29-0.63)$. Table 4 gives the information of the model summary for the artificial neural network. The sum of square error was 0.722 for training by five consecutive step(s) and 2.873 for testing. That means the accuracy of the ANN model was $100 \%$ and $82.4 \%$ for training and testing, respectively. Supplemental Table 1 gives the information about parameter estimates for the artificial neural network to build the ANNs model. The predictor is used in this table as input variables and the two hidden layers. The output layer indicated by applicator type ' 0 ' means tri-channel applicator, and ' 1 ' means singlechannel applicator. Table 5 shows the confusion matrix for the artificial neural network of the singlechannel applicator and tri-channel applicator. The sensitivity, specificity, and accuracy were $100 \%, 100 \%$, and $100 \%$ for training, while $87.5 \%, 77.8 \%$, and $82.4 \%$ for testing.

Fig. 4. describes the pseudo-probability for the single-channel applicator and tri-channel applicator in cervical cancer. It explains the clustered box-plot for combined the training and testing samples based on categorical dependent variables such as tri-channel applicator and single-channel applicator. The $\mathrm{x}$-axis indicates observed response categories, and the legend makes predicted categories. The leftmost first box-plot shows the observed category tri-channel applicator for cases and has the predicted pseudo probability of the type of tri-channel applicator. The second box plot shows the observed category trichannel applicator, but the predicted pseudo probability of the category tri-channel applicator. The third box-plot for patients exits the empirical category single channel application while the predicted pseudo probability is the category tri-channel applicator. Lastly, the fourth box plot for cases shows the observed category single channel with the predicted pseudo probability of category single channel.

Fig. 5. depicts the sensitivity and specificity based on the single-channel applicator and tri-channel applicator for cervical cancer, demonstrating the display of sensitivity and specificity at cutoffs for training and testing samples. The area under the curve was 0.961 for the single-channel applicator and tri-channel applicator outcomes. Fig. 6 . shows the gain of a single-channel applicator and tri-channel 
applicator for cervical cancer. For example, in the first point is for the tri-channel applicator, it was found (10\%, $25 \%$ ), demonstrating that we expect the top $10 \%$ to contain the approximate $25 \%$ of all cases that take the category tri-channel applicator if we score the data set with the neural network and sort all of the cases by predicted pseudo probability of tri-channel applicator. Similarly, the top $20 \%$ would contain the approximate $45 \%$ of the defaulters, the top of $30 \%$ cases, $70 \%$ of defaulters, and so on. If we select $100 \%$ of the scored data, we will obtain all data of the defaulters for single-channel and tri-channel applicator. Fig. 7. shows the lift curve for the single-channel applicator and tri-channel applicator for cervical cancer. The value of the $y$-axis represents the ratio of the cumulative gain for each curve to the baseline. Thus, the lift at $10 \%$ for the tri-channel applicator is $25 \% / 10 \%=2.5$ and $21 \% / 10 \%=2.1$ for single-channel applicator based on Fig. 6. Fig. 8. indicates the normalization importance of single-channel applicator and tri-channel applicator in cervical cancer. It describes that Ovid length was the highest predictor and EBRT was the lowest predictor based on normalization. Fig. 9. shows the follow-up time of the survival rate based on a single channel and tri-channel applicator's treatment outcomes. At two years, the survival rate for a single-channel was $85 \%$, which was lower than $95 \%$ for a tri-channel applicator.

\section{Discussion}

In this research, the cervical cancer data has been used to build up an ANN model, demonstrating a neural network that has an input layer, hidden layer, and an output layer stated the treatment outcomes for single-channel and tri-channel applicators, activation function identity, and provided the sensitivity and specificity with superior accuracy. The model also described the pseudo-probability, gain, and lift curve with area under the curve (AUC) for single-channel and tri-channel applicator of cervical cancer. The treatment outcomes measured based on survival analysis stated the tri-channel applicator has a higher potential than the single-channel applicator.

Many researchers have used the ANN model to predict the model's based clinical data. Wang et al.[20] proposed an ANN model for traumatic brain injury, demonstrating the prediction of hematoma based on age, bone flop size, glucose level, pupillary response, and the overall accuracy was $73.0 \%$. Azimi et al.[21] reported that the ANN model was established with an accuracy of $96.9 \%$ and a better ROC value of $80 \%$ for lumbar spinal canal stenosis. Tang et al.[25] used the back prorogation algorithm by artificial neural network for Alzheimer disease screening, resulting in the sensitivity, specificity, and accuracy of $90 \%, 95 \%$, and $92.50 \%$, respectively. Bottaci et al.[22] suggested that the ANN model for colorectal cancer patients described the sensitivity, specificity, and accuracy of $66 \%, 88 \%$, and $80 \%$, respectively. Baxt et al.[24] suggested an ANN model for myocardial infection, resulting in the sensitivity and specificity were $97.2 \%$ and $96.2 \%$. We found in our study that the model accuracy performance was superior to judge the treatment outcomes used by the applicators in cervical cancer. In the current study, the accuracy was $100 \%$, and $82.4 \%$ for the training and testing included AUC $=0.961$, respectively, in the present study. The sensitivity and specificity were $100 \%$ and $100 \%$ for training and $87.5 \%$, and $77.8 \%$ for testing.

An ANN model has the potential power to predict the risk factor analysis according to the American Society of Anesthesiology (ASA) class $>3$ for posterior lumbar spine fusion that has been reported in Kim 
et al.[23] For cervical cancer, Jaberi et al.[18] proposed image-guided brachytherapy for treatment plan correction of OARs in intra-fraction organ, suggesting the final brachytherapy treatment plan modified based on changed the organ applicators to compensate the target dose controlled at the original level. In chronic lymphocytic leukemia, Aghamaleki et al.[26] proposed an ANN model to detect the molecular biomarker for cancer diagnosis from blood samples. The survival rate studied for gastric cancer patients in Charati et al.[27] The median survival rate was $19 \pm 2.04$ months at five years, demonstrating an AUC of $94 \%$ based on factors such as stage of diseases, metastasis, histology grade, and age of diagnosis. The treatment outcomes based on survival rate were $91.6 \%$ and $89.4 \%$ for Co-60 and Ir-192 at 2-years in the literature in stages Ib2-111b of cervical cancer.[28] Li et al.[29] have reported that the survival rate for high dose rate brachytherapy for the fletcher group and single-channel group was $80.3 \%$ and $86.3 \%$ in cervical cancer at 2-years. In our study, at 2-years, the survival rate was $85 \%$ and $95 \%$ for the singlechannel applicator and tri-channel applicator, respectively. Pang et al.[30] reported an ANN model for the pathological voice by quantities analysis and detection, suggesting the higher accuracy for identification with good clinical information. Li et al.[31] proposed an ANN model to predict the risk factor for heart disease in congenital heart disease, suggesting the sensitivity, specificity was $87 \%$ and $90 \%$ for the training set. The AUC value of training and test set were 0.87 and 0.97 , respectively. Kuang et al.[32] proposed an ANN model for Alzheimer's disease, describing the sensitivity, specificity, and AUC were $82.11 \pm 0.42 \%, 75.26 \pm 0.86 \%$, and $92.08 \pm 0.12 \%$, respectively, included accuracy $89.52 \pm 0.36 \%$. In our study, the AUC value was 0.961 .

Rajković et al.[17] suggested an ANN model for the treatment of prostate carcinoma, resulting in the therapy dose (TD) of 47.3 Gy and coverage index (Cl100\%) of 1.4 for the low-risk group and TD of 50.4 Gy and $\mathrm{Cl} 100 \% 1.6$ for the high-risk group. In this research, we treated better therapy doses for cervical cancer patients to build up the ANNs model. The EBRT was 45 (40-50) cGy after chemotherapy. During the period of brachytherapy, the rectum dose and bladder dose were in the following: Rectum Dose 3.71 (2.29-5.14) Gy; Bladder Dose 2 (1.94-5.83) Gy and Prescribe Dose 6.87 (6-7) Gy. We found Tumor Area Dose 6.74 (5.64-7) Gy and Dose Volume $113.6(58.2-469.25) \mathrm{cm}^{3}$.

There are some limitations. The ANNs can identify the complex and non-linear relationship between independent and dependent variables and detect all possible interactions for all predictors.[33] The ANNs have some disadvantages. The 'Black Box' cannot have explained the odd ratio that identifies the direction and magnitude of the effect of each variable like LR.[34] The ANNs model is prone to adjust the overfitting data that the model is not perfect for generalization to the external data.[33] The optimization problem of the ANNs model is complex, such as training times, several nodes, regulations, and layers to proceed optimally the outcomes.[35]

\section{Conclusion}

An artificial neural network established for promising methods for patient treatments. The network built based on clinical data for cervical cancer using a single channel applicator and tri-channel applicator. A multi-layer preconception was trained with a backpropagation algorithm, demonstrating the accuracy of 
$100 \%$ for training and $82.4 \%$ for testing with $A U C=0.961$. The proposed model can evaluate the treatment outcomes for single-channel and tri-channel applicators in cervical cancer based on survival analysis after the last follow-up.

\section{Declarations}

\section{Conflict of interest}

The authors declare that they have no conflict of interest.

\section{Ethical approval}

The study was approved by a human research ethics committee, Rajshahi Medical College and Hospital, Bangladesh

Disclosure / Conflict of Interest Statement: None

\section{References}

1. Fowler JR, Jack BW (2021) Cervical Cancer. In: StatPearls. StatPearls Publishing, Treasure Island $(\mathrm{FL})$

2. Olusola P, Banerjee HN, Philley JV, Dasgupta S (2019) Human papilloma virus-associated cervical cancer and health disparities. Cells 8:622

3. Gaffney DK, Hashibe M, Kepka D, et al (2018) Too many women are dying from cervix cancer:

Problems and solutions. Gynecol Oncol 151:547-554. https://doi.org/10.1016/j.ygyno.2018.10.004

4. Ferlay J, Soerjomataram I, Dikshit R, et al (2015) Cancer incidence and mortality worldwide: sources, methods and major patterns in GLOBOCAN 2012. Int J Cancer 136:E359-386. https://doi.org/10.1002/ijc.29210

5. Vaccarella S, Lortet-Tieulent J, Plummer M, et al (2013) Worldwide trends in cervical cancer incidence: impact of screening against changes in disease risk factors. Eur J Cancer 49:3262-3273. https://doi.org/10.1016/j.ejca.2013.04.024

6. Yang $L$, Yang $Y$, Meng $M$, et al (2021) Identification of prognosis-related genes in the cervical cancer immune microenvironment. Gene 766:145119. https://doi.org/10.1016/j.gene.2020.145119

7. Viswanathan AN, Beriwal S, Jennifer F, et al (2012) American Brachytherapy Society consensus guidelines for locally advanced carcinoma of the cervix. Part II: high-dose-rate brachytherapy. Brachytherapy 11:47-52

8. Demanes DJ, Rege S, Rodriquez RR, et al (1999) The use and advantages of a multichannel vaginal cylinder in high-dose-rate brachytherapy. International Journal of Radiation Oncology* Biology* Physics 44:211-219 
9. Park S-J, Chung M, Demanes DJ, et al (2013) Dosimetric comparison of 3-dimensional planning techniques using an intravaginal multichannel balloon applicator for high-dose-rate gynecologic brachytherapy. International Journal of Radiation Oncology* Biology* Physics 87:840-846

10. Nick TG, Campbell KM (2007) Logistic regression. Topics in biostatistics 273-301

11. Harrell Jr FE, Lee KL, Mark DB (1996) Multivariable prognostic models: issues in developing models, evaluating assumptions and adequacy, and measuring and reducing errors. Statistics in medicine 15:361-387

12. Patel JL, Goyal RK (2007) Applications of artificial neural networks in medical science. Current clinical pharmacology 2:217-226

13. Stergiou C, Siganos D. Neural Networks (2017): Imperial College of London, Computer Department Available at: http://www.doc.ic.ac.uk/ nd/surprise_96/journal/vol4/cs11/report.html\#Contents.

14. Miller S, Bews J, Kinsner W (2001) Brachytherapy cancer treatment optimization using simulated annealing and artificial neural networks. In: Canadian Conference on Electrical and Computer Engineering 2001. Conference Proceedings (Cat. No. 01TH8555). IEEE, pp 649-654

15. Cross SS, Harrison RF, Kennedy RL (1995) Introduction to neural networks. The Lancet 346:10751079

16. Gao J, Zagadailov P, Merchant AM (2021) The Use of Artificial Neural Network to Predict Surgical Outcomes After Inguinal Hernia Repair. Journal of Surgical Research 259:372-378

17. Rajković KM, Dabić-Stanković K, Stanković J, et al (2020) Modelling and optimisation of treatment parameters in high-dose-rate mono brachytherapy for localised prostate carcinoma using a multilayer artificial neural network and a genetic algorithm: Pilot study. Comput Biol Med 126:104045. https://doi.org/10.1016/j.compbiomed.2020.104045

18. Jaberi R, Siavashpour Z, Aghamiri MR, et al (2017) Artificial neural network based gynaecological image-guided adaptive brachytherapy treatment planning correction of intra-fractional organs at risk dose variation. J Contemp Brachytherapy 9:508-518. https://doi.org/10.5114/jcb.2017.72567

19. Widrow B, Rumelhart DE, Lehr MA (1994) Neural networks: applications in industry, business and science. Communications of the ACM 37:93-106

20. Wang J-L, Jin G-L, Yuan Z-G (2021) Artificial neural network predicts hemorrhagic contusions following decompressive craniotomy in traumatic brain injury. J Neurosurg Sci 65:69-74. https://doi.org/10.23736/S0390-5616.17.04123-6

21. Azimi P, Benzel EC, Shahzadi S, et al (2014) Use of artificial neural networks to predict surgical satisfaction in patients with lumbar spinal canal stenosis. Journal of Neurosurgery: Spine 20:300305

22. Bottaci L, Drew PJ, Hartley JE, et al (1997) Artificial neural networks applied to outcome prediction for colorectal cancer patients in separate institutions. The Lancet 350:469-472

23. Kim JS, Merrill RK, Arvind V, et al (2018) Examining the ability of artificial neural networks machine learning models to accurately predict complications following posterior lumbar spine fusion. Spine 43:853 
24. Baxt WG (1991) Use of an artificial neural network for the diagnosis of myocardial infarction. Annals of internal medicine 115:843-848

25. Tang J, Wu L, Huang H, et al (2013) Back propagation artificial neural network for community Alzheimer's disease screening in China. Neural Regen Res 8:270-276. https://doi.org/10.3969/j.issn.1673-5374.2013.03.010

26. Aghamaleki FS, Mollashahi B, Nosrati M, et al (2019) Application of an artificial neural network in the diagnosis of chronic lymphocytic leukemia. Cureus 11:

27. Charati JY, Janbabaei G, Alipour N, et al (2018) Survival prediction of gastric cancer patients by Artificial Neural Network model. Gastroenterology and hepatology from bed to bench 11:110

28. Tantivatana T, Rongsriyam K (2018) Treatment outcomes of high-dose-rate intracavitary brachytherapy for cervical cancer: a comparison of Ir-192 versus Co-60 sources. J Gynecol Oncol 29:. https://doi.org/10.3802/jgo.2018.29.e86

29. Li D, Wen E, Zhang Y, et al (2018) Preliminary report of a single-channel applicator in high dose rate afterloading brachytherapy for cervical cancer. Cancer science 109:3953-3961

30. Pang YF, Huang J, Xu BZ, et al (2016) Quantitative analysis of pathological voice and identification with artificial neural network. Lin Chung Er Bi Yan Hou Tou Jing Wai Ke Za Zhi 31:100-102. https://doi.org/10.13201/j.issn.1001-1781.2017.02.005

31. Li H, Luo M, Zheng J, et al (2017) An artificial neural network prediction model of congenital heart disease based on risk factors: A hospital-based case-control study. Medicine (Baltimore) 96:e6090. https://doi.org/10.1097/MD.0000000000006090

32. Kuang J, Zhang P, Cai T, et al (2021) Prediction of transition from mild cognitive impairment to Alzheimer's disease based on a logistic regression-artificial neural network-decision tree model. Geriatr Gerontol Int 21:43-47. https://doi.org/10.1111/ggi.14097

33. Tu JV (1996) Advantages and disadvantages of using artificial neural networks versus logistic regression for predicting medical outcomes. J Clin Epidemiol 49:1225-1231. https://doi.org/10.1016/s0895-4356(96)00002-9

34. Benitez JM, Castro JL, Requena I (1997) Are artificial neural networks black boxes? IEEE Trans Neural Netw 8:1156-1164. https://doi.org/10.1109/72.623216

35. Benardos PG, Vosniakos G-C (2007) Optimizing feedforward artificial neural network architecture. Engineering applications of artificial intelligence 20:365-382

\section{Tables}

\section{Table 1. Clinical characteristics of cervical cancer patients}




\begin{tabular}{|llllllllllll|}
\hline $\begin{array}{l}\text { Age } \\
\text { (years) }\end{array}$ & $\begin{array}{l}\text { Number of } \\
\text { cases }\end{array}$ & Training & Testing & \multicolumn{2}{c|}{ Histopathology } & \multicolumn{5}{c|}{ Staging of cervical cancer } \\
\hline $50(28-75)$ & 54 & 37 & 17 & 51 & 3 & 3 & 8 & 27 & 16 & 0 \\
\hline
\end{tabular}

sqca: Squamous cell carcinoma; adca: adenocarcinoma

Table 2: Summary of cases for the artificial neural network

\begin{tabular}{|llll|}
\hline & & N & Percent \\
\hline Sample & Training & 37 & $68.50 \%$ \\
\hline & Testing & 17 & $31.50 \%$ \\
\hline Valid & & 54 & $100.00 \%$ \\
\hline Excluded & & 0 & \\
\hline Total & & 54 & \\
\hline
\end{tabular}

Table 3 Network Information to build up the artificial neural network 


\begin{tabular}{|c|c|c|c|}
\hline \multirow[t]{22}{*}{ Input Layer } & \multirow[t]{20}{*}{ Covariates } & 1 & Age (years): \\
\hline & & 2 & EBRT(cGy): \\
\hline & & 3 & $5.56(0.47-0.76)$ \\
\hline & & 4 & Air Kerma Strength $\left(\mathrm{cGycm}^{2} \mathrm{~h}\right)$ : \\
\hline & & & $13865.52(11158.5-57390.0)$ \\
\hline & & 5 & Activity (Ci): $\quad 1.17(0.99-1.59)$ \\
\hline & & 6 & Dose Volume $\left(\mathrm{cm}^{3}\right) 113.6(58.2-469.25)$ \\
\hline & & 7 & Applicator Insertion (cm): $4.78(2-8)$ \\
\hline & & 8 & Ovid Length (mm): $24.72(15-30)$ \\
\hline & & 9 & Treatment Duration (days): 13.26 (6-27) \\
\hline & & 10 & Treatment Fraction: \\
\hline & & 11 & Treatment Results: $\quad 0.46(0-1)$ \\
\hline & & 12 & Alive/ Dead: \\
\hline & & 13 & Prescribe Dose (Gy): $\quad 6.87(6-7)$ \\
\hline & & 14 & Tumor Area Dose (Gy): 6.74 (5.64-7) \\
\hline & & 15 & Manchester B (Gy): $\quad 1,85$ (1.50-2.65) \\
\hline & & 16 & Rectum Dose (Gy): 3.71 (2.29-5.14) \\
\hline & & 17 & Bladder Dose.(Gy): $\quad 2$ (1.94-5.83) \\
\hline & & 18 & Treatment Time (min): \\
\hline & & 19 & TRAK (cGy.m²): $0.43(0.29-0.63)$ \\
\hline & \multicolumn{2}{|l|}{ Number of Units ${ }^{a}$} & 19 \\
\hline & \multicolumn{2}{|l|}{$\begin{array}{l}\text { Rescaling Method for } \\
\text { Covariates }\end{array}$} & Adjusted normalized \\
\hline \multirow{4}{*}{$\begin{array}{l}\text { Hidden } \\
\text { Layer(s) }\end{array}$} & \multicolumn{2}{|l|}{ Number of Hidden Layers } & 2 \\
\hline & \multicolumn{2}{|l|}{$\begin{array}{l}\text { Number of Units in Hidden } \\
\text { Layer } 1^{\mathrm{a}}\end{array}$} & 10 \\
\hline & \multicolumn{2}{|l|}{$\begin{array}{l}\text { Number of Units in Hidden } \\
\text { Layer } 2^{\mathrm{a}}\end{array}$} & 8 \\
\hline & \multicolumn{2}{|l|}{ Activation Function } & Hyperbolic tangent \\
\hline Output & Dependent Variables & 1 & Treatment Type: Single channel applicator or tri- \\
\hline
\end{tabular}


Number of Units

Activation Function

Error Function
2

Identity

Sum of Squares

a. Excluding the bias unit

EBRT: External beam radiation therapy; TRAK: Total reference air kerma.

Table 4 Summary of the proposed model for the artificial neural network

\begin{tabular}{|cll|}
\hline \multirow{3}{*}{ Training } & Sum of Squares Error & .722 \\
\cline { 2 - 3 } & Percent Incorrect Predictions & $0.0 \%$ \\
\cline { 2 - 3 } & Stopping Rule Used & 5 consecutive step(s) with no decrease in error $^{\mathrm{a}}$ \\
\cline { 2 - 3 } Testing & Training Time & $0: 00: 00.00$ \\
\cline { 2 - 3 } & Sum of Squares Error & 2.873 \\
\cline { 2 - 3 } & Percent Incorrect Predictions & $17.6 \%$ \\
\hline
\end{tabular}

Dependent Variable: Treatment Type (Single Channel applicator/Tri-channel applicator)

a. Error computations are based on the testing sample.

Table 5 Confusion matrix for single channel applicator and tri-channel applicator in the the artificial neural networks

\begin{tabular}{|lllll|}
\hline Sample & Observed & \multicolumn{3}{l|}{ Predicted } \\
\cline { 3 - 5 } & & .0 & 1.0 & Percent Correct \\
\hline \multirow{3}{*}{ Training } & .0 & 17 & 0 & $100.0 \%$ \\
\cline { 2 - 5 } & 1.0 & 0 & 20 & $100.0 \%$ \\
\cline { 2 - 5 } Testing & Overall Percent & $45.9 \%$ & $54.1 \%$ & $100.0 \%$ \\
& .0 & 7 & 1 & $87.5 \%$ \\
\cline { 2 - 5 } & 1.0 & 2 & 7 & $77.8 \%$ \\
\cline { 2 - 5 } & Overall Percent & $52.9 \%$ & $47.1 \%$ & $82.4 \%$ \\
\hline
\end{tabular}

Tri channel applicator $=0$, Single channel applicator $=1$ 
Figures

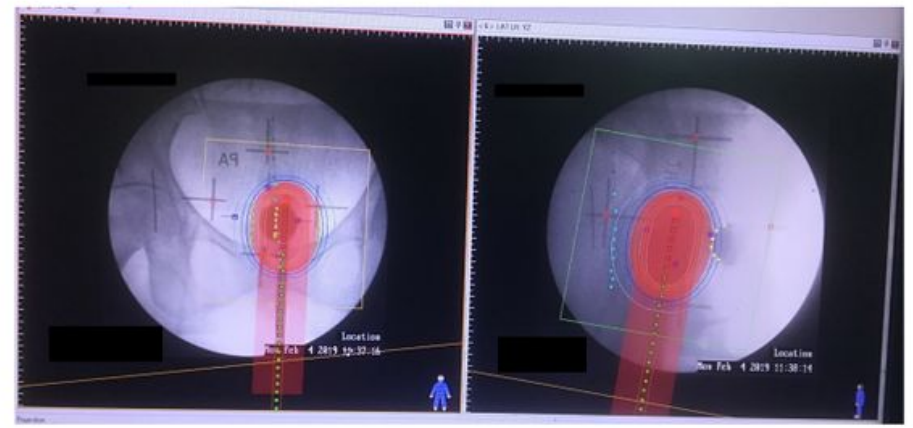

(a)

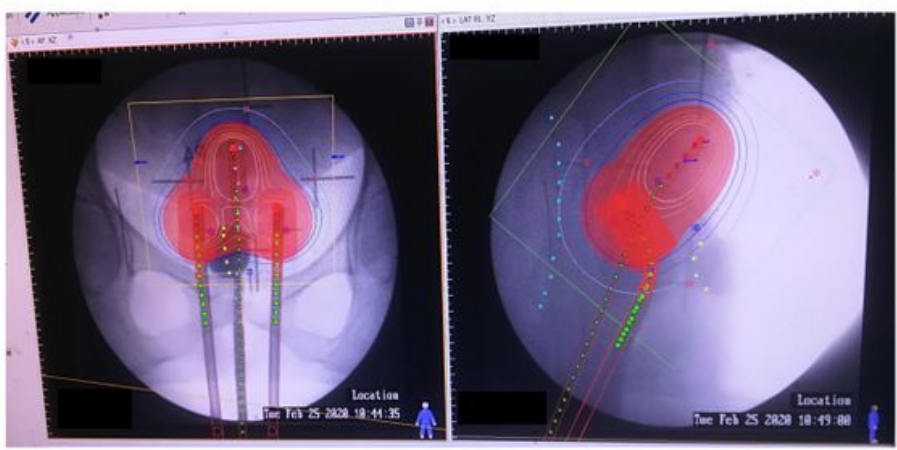

(b)

Single Channel

Tri- Channel

\section{Figure 1}

Dose distribution for AP (a) and lateral (b) view for single-channel and tri-channel applicator 

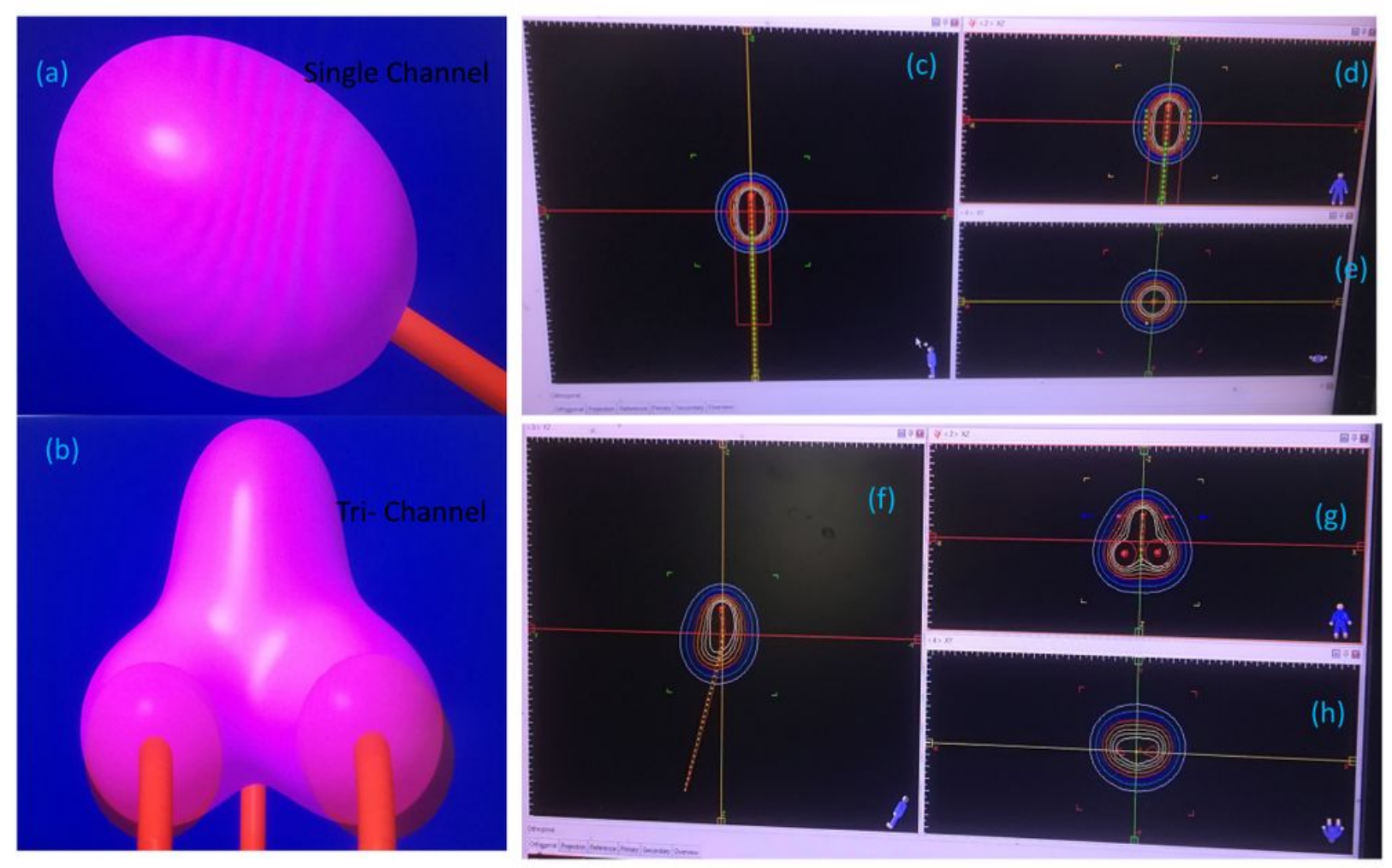

Figure 2

Graphical representation of (a) single-channel and (b) tri-channel applicator; Dose distribution in (c) axial, (d), sagittal, (e) coronel for the single-channel applicator and dose distribution in (f) axial, (g), sagittal, (h) coronel for the tri-channel applicator 


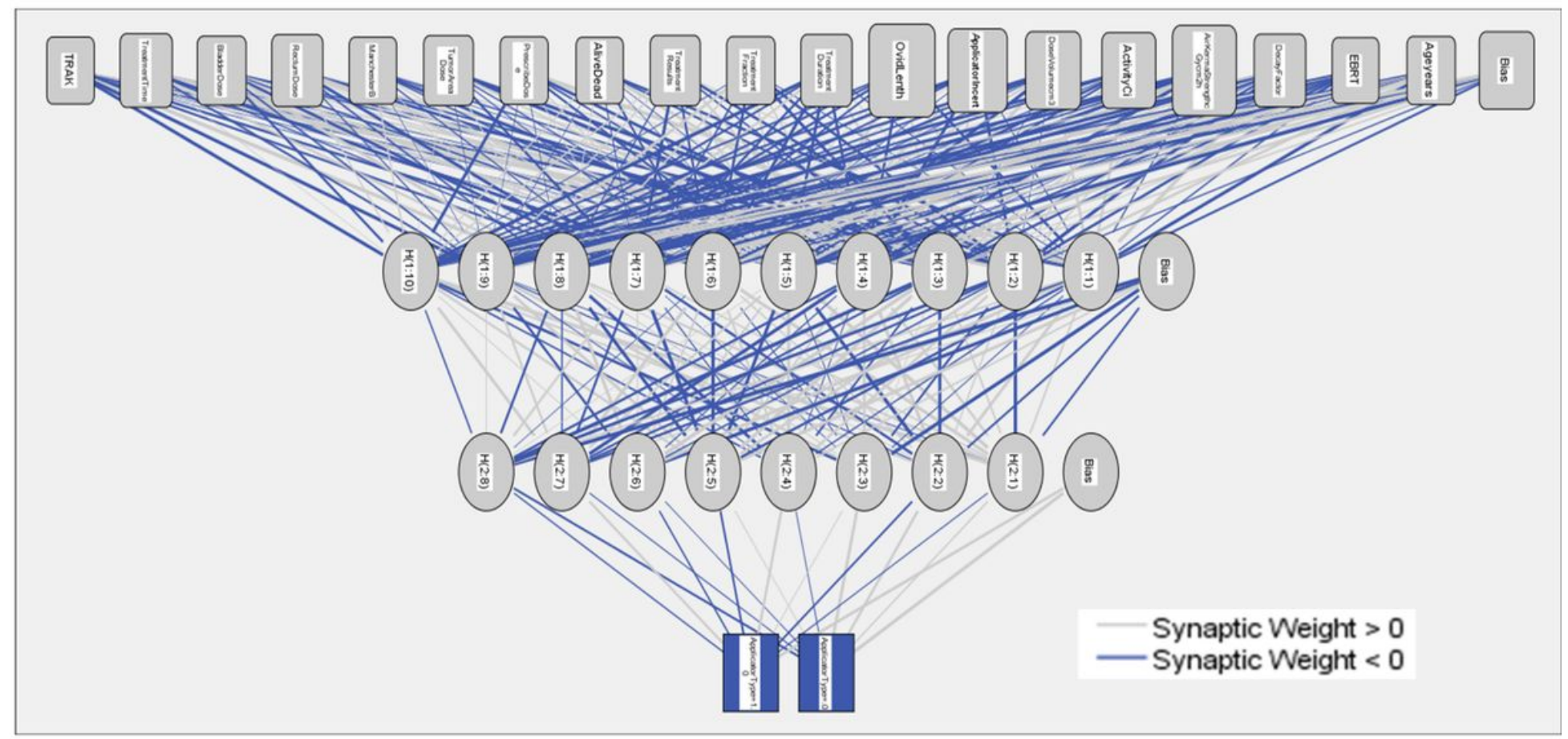

\section{Figure 3}

Artificial Neural network for two treatment types of cervical cancer (applicator=0, applicator=1); hidden layer activation function: Hyperbolic tangent; Output layer activation function: identity.

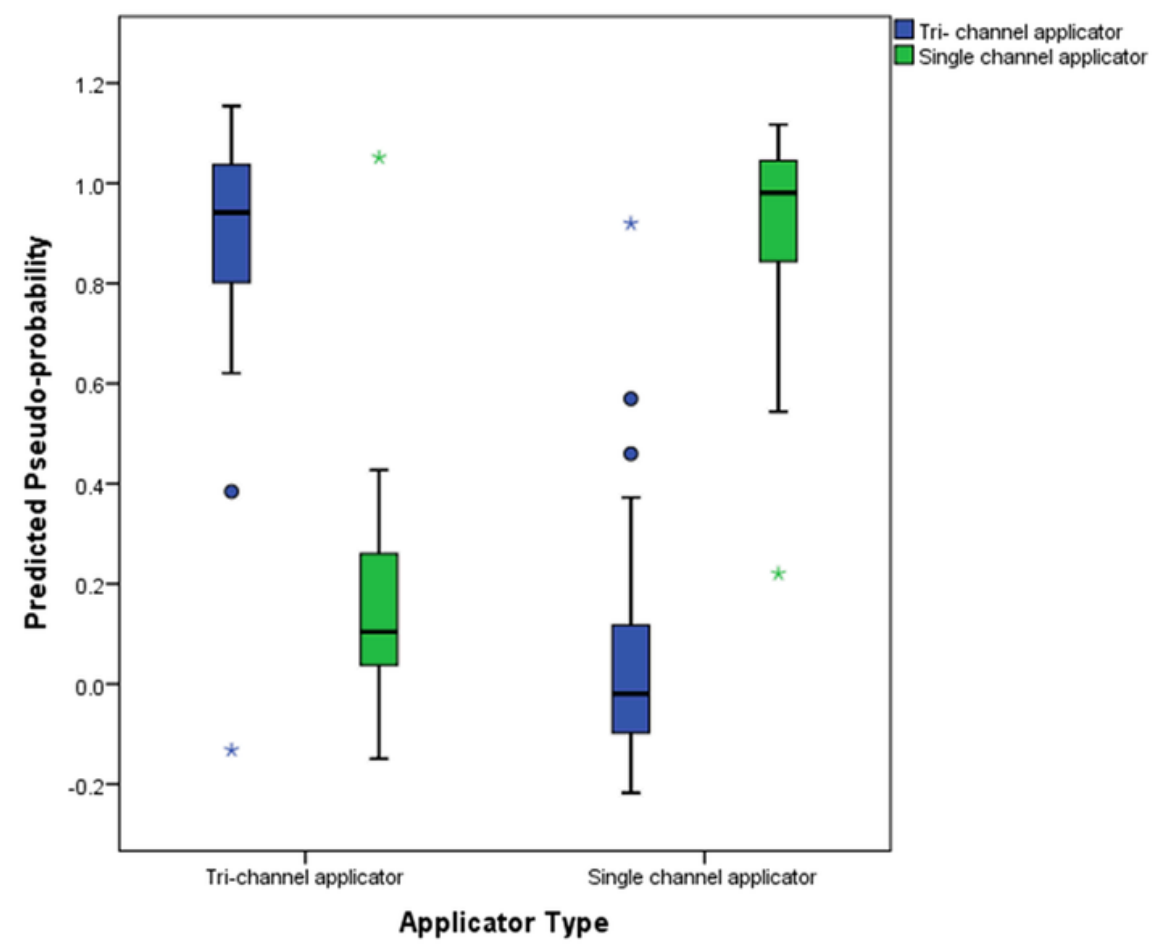




\section{Figure 4}

The pseudo-probability curve for the single-channel applicator and tri-channel applicator for cervical cancer

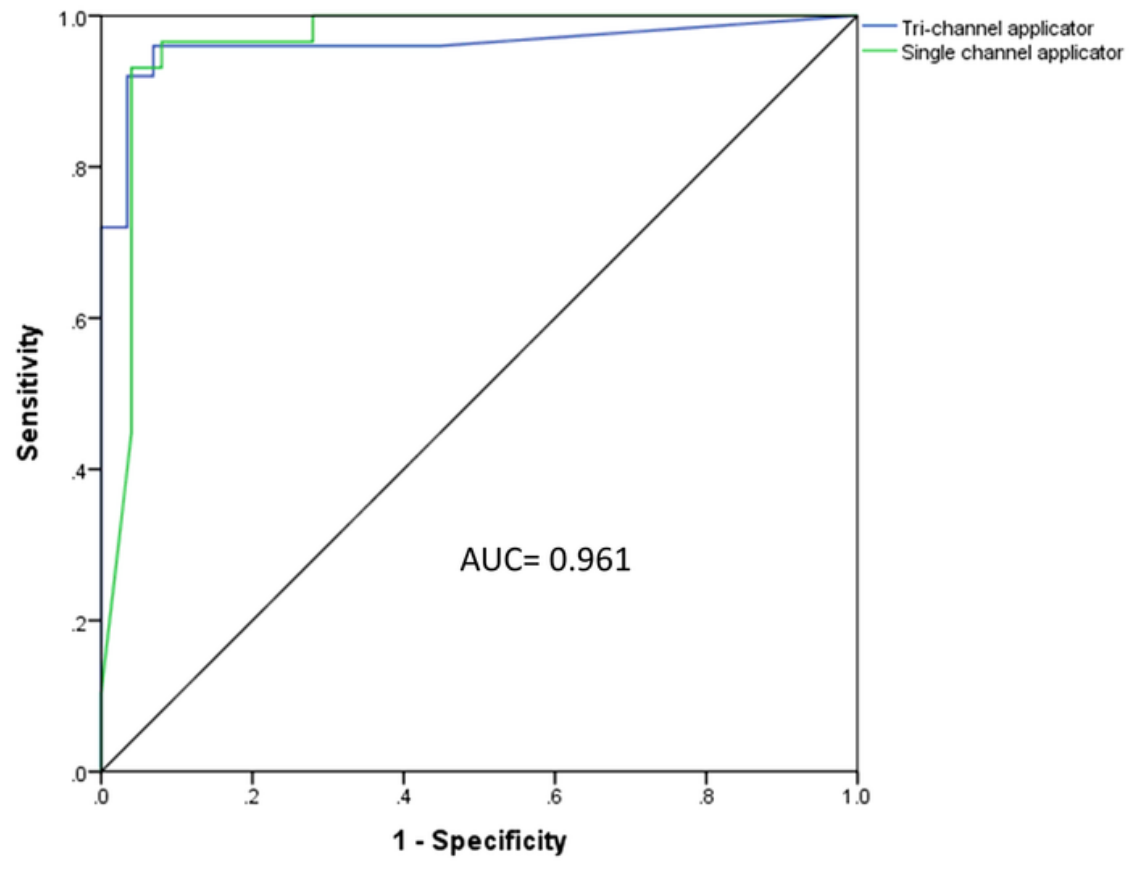

\section{Figure 5}

Sensitivity and specificity curve for the single-channel applicator and tri-channel applicator for cervical cancer 


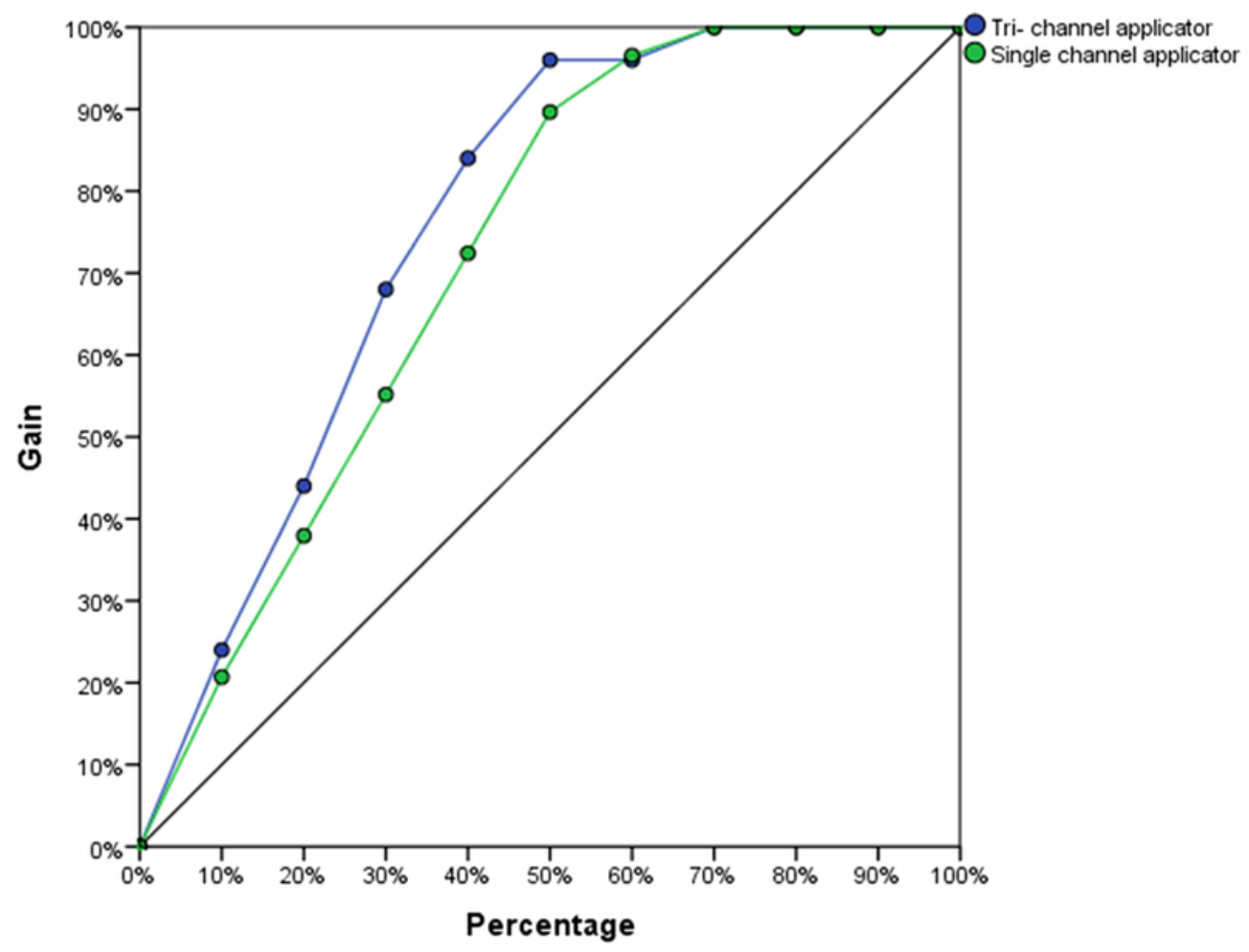

Figure 6

The gain curve for the single-channel applicator and tri-channel applicator for cervical cancer

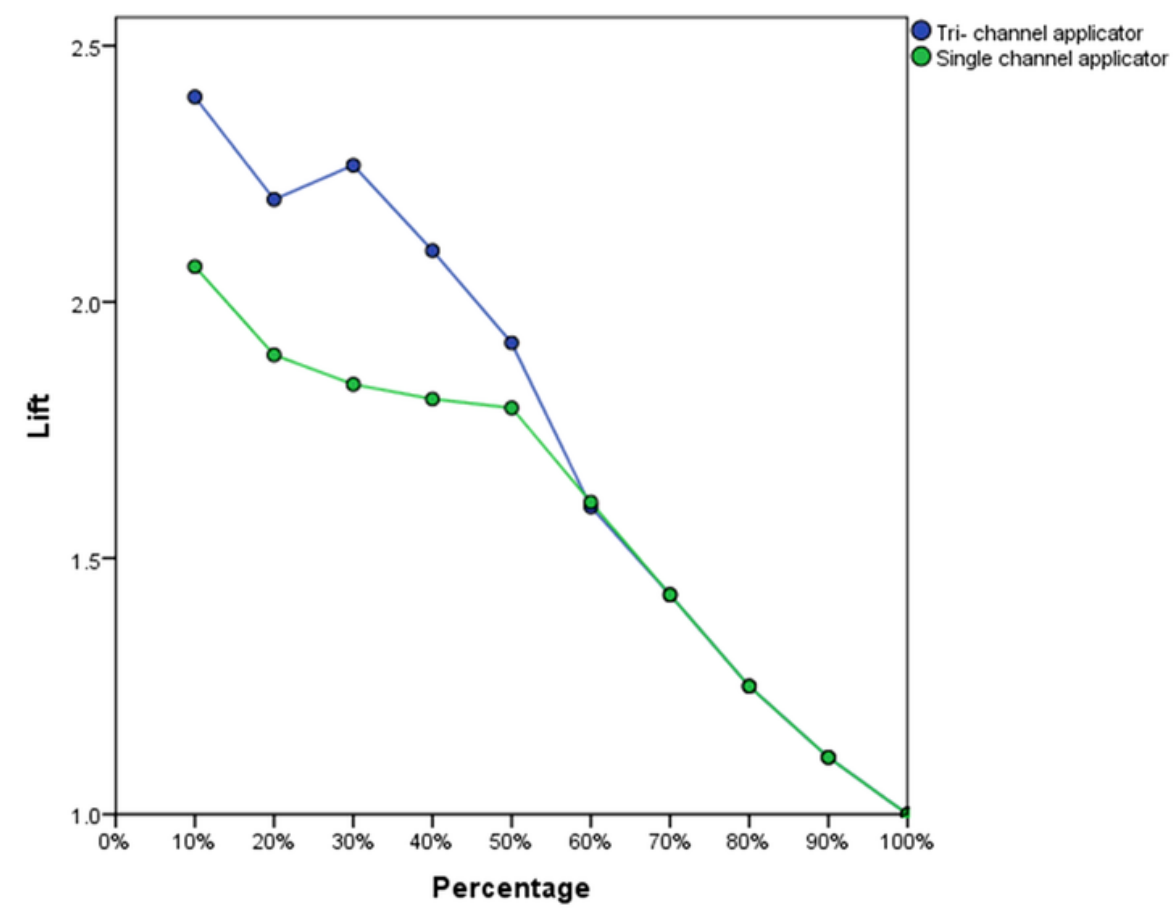


Figure 7

The Lift curve for the single-channel applicator and tri-channel applicator for cervical cancer.

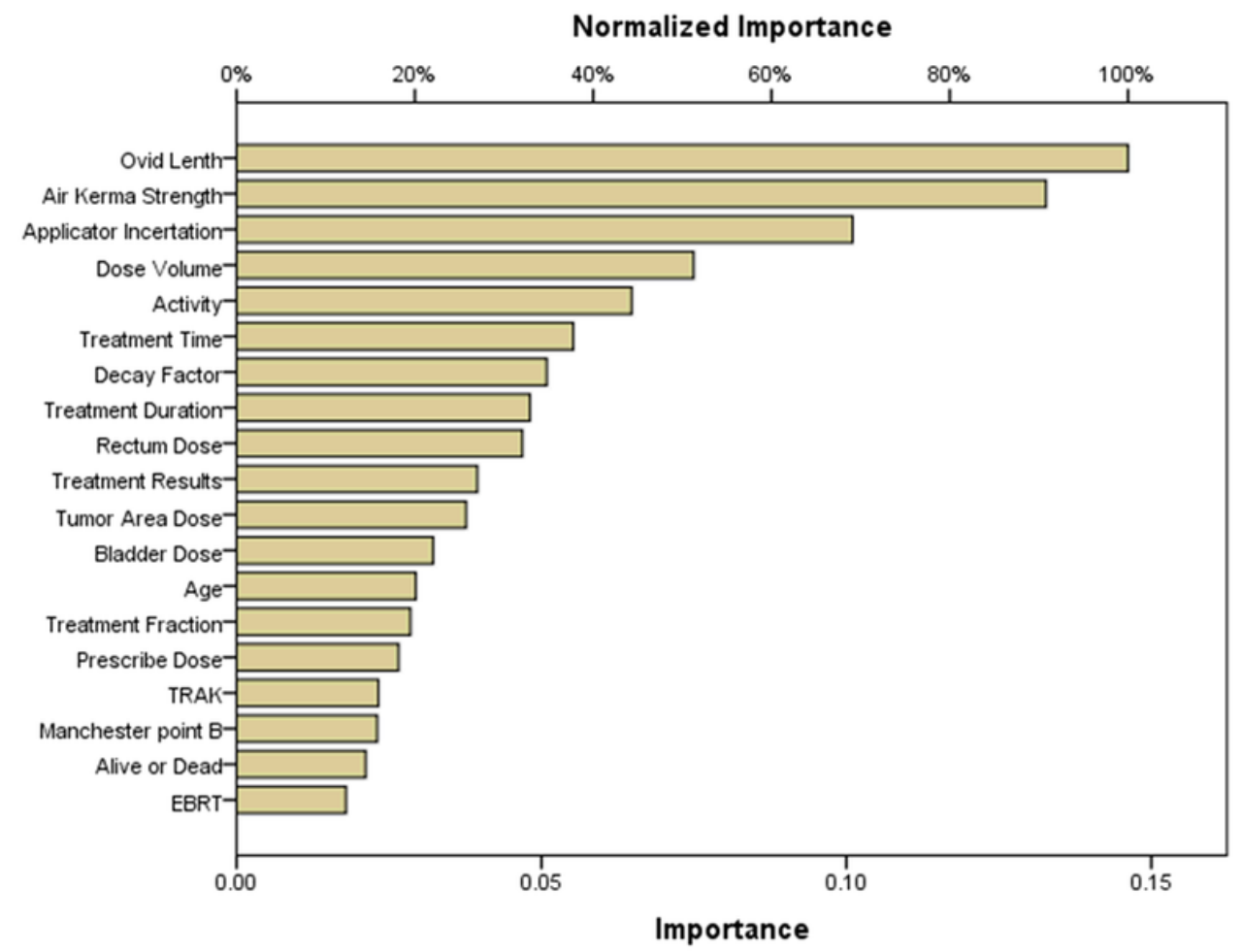

\section{Figure 8}

Normalization importance curve for the single-channel applicator and tri-channel applicator for cervical cancer 


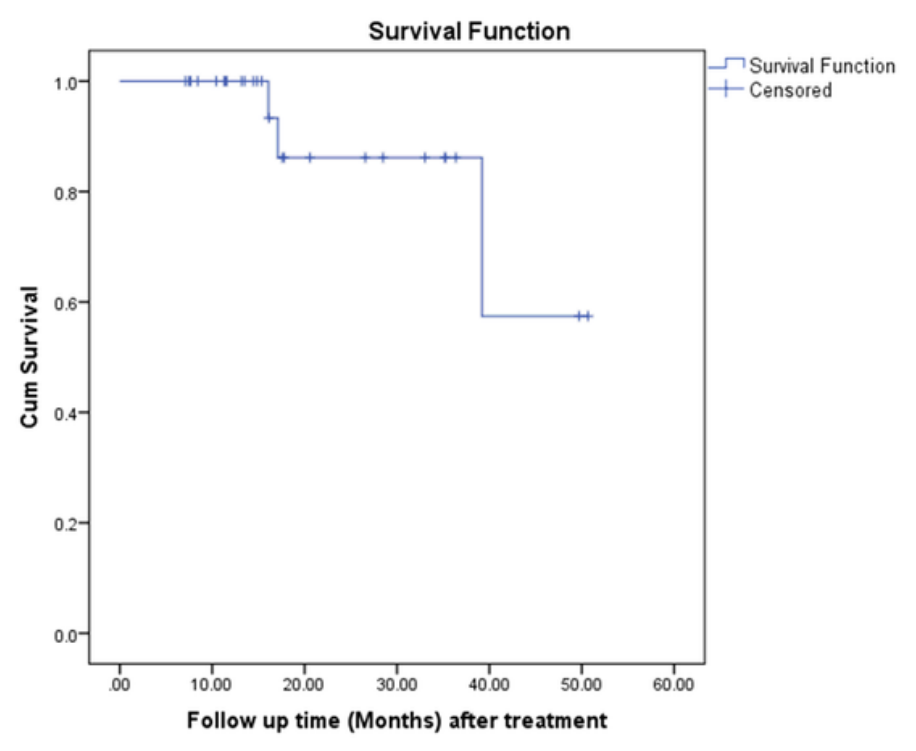

(a)

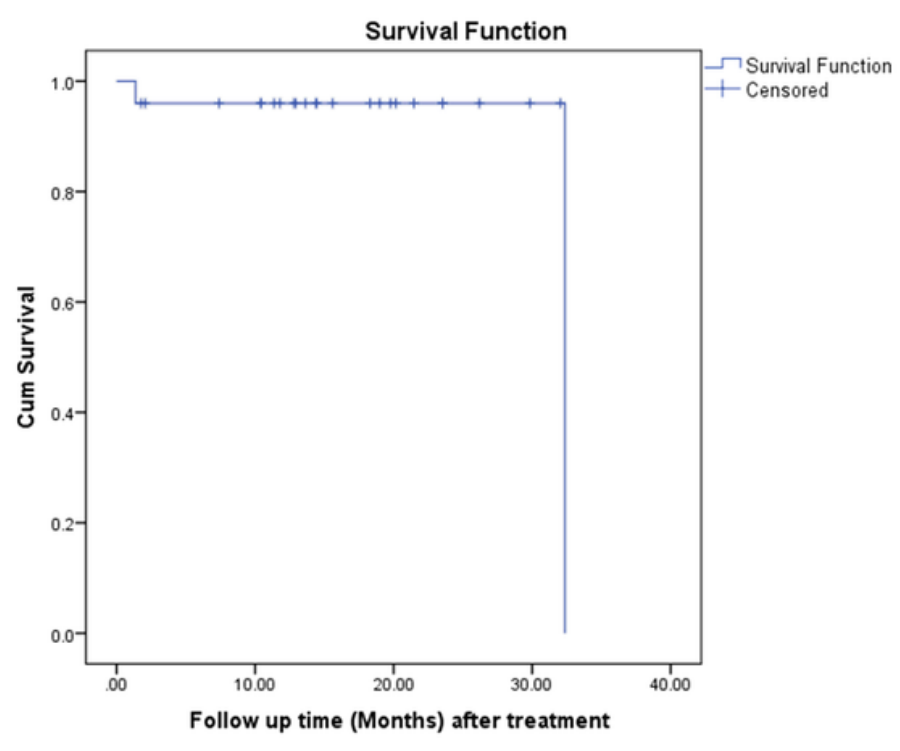

(b)

\section{Figure 9}

Follow-up time (time) after treatment based on survival analysis for (a) single-channel applicator and (b) tri-channel applicator. 